\title{
The impact of preoperative patient characteristics on health states after total hip replacement and related satisfaction thresholds: a cohort study
}

\author{
Matthias Vogl ${ }^{1,2^{*}}$, Rainer Wilkesmann ${ }^{3}$, Christian Lausmann ${ }^{3}$, Matthias Hunger ${ }^{1}$ and Werner Plötz ${ }^{3,4}$
}

\begin{abstract}
Background: The aim of the study was to analyze the effect of preoperative patient characteristics on health outcomes 6 months after total hip replacement (THR), to support patient's decision making in daily practice with predicted health states and satisfaction thresholds. By giving incremental effects for different patient subgroups, we support comparative effectiveness research (CER) on osteoarthritis interventions.

Methods: In 2012, 321 patients participated in health state evaluation before and 6 months after THR. Health-related quality of life (HRQoL) was measured with the EQ-5D questionnaire. Hip-specific pain, function, and mobility were measured with the WOMAC in a prospective observation of a cohort. The predictive capability of preoperative patient characteristics - classified according to socio-demographic factors, medical factors, and health state variables - for changes in health outcomes is tested by correlation analysis and multivariate linear regressions. Related satisfaction thresholds were calculated with the patient acceptable symptom state (PASS) concept.
\end{abstract}

Results: The mean WOMAC and EQ-5D scores before operation were 52 and 60 respectively ( 0 worst, 100 best). At the 6-month follow-up, scores improved by 35 and 19 units. On average, patients reported satisfaction with the operation if postoperative (change) WOMAC scores were higher than 85 (32) and postoperative (change) EQ-5D scores were higher than 79 (14).

Conclusions: Changes in WOMAC and EQ-5D scores can mainly be explained by preoperative scores. The lower the preoperative WOMAC or EQ-5D scores, the higher the change in the scores. Very good or very poor preoperative scores lower the probability of patient satisfaction with THR. Shared decision making using a personalized risk assessment approach provides predicted health states and satisfaction thresholds.

Keywords: Health-related quality of life, EQ-5D, WOMAC, Total hip replacement, Satisfaction, Patient acceptable symptom state

\section{Background}

Total hip replacement (THR) is an effective operation that relieves pain and improves function, mobility, and health-related quality of life (HRQoL) in patients with osteoarthritis and further diagnoses [1-4]. As hip replacement rates are increasing greatly worldwide $[5,6]$,

\footnotetext{
* Correspondence: matthias.vog|@helmholtz-muenchen.de

${ }^{1}$ Helmholtz Zentrum München, German Research Center for Environmental Health, Institute of Health Economics and Health Care Management, P.O. Box 1129, Neuherberg 85758, Germany

${ }^{2}$ Ludwig-Maximilians-Universität München, Munich School of Management, Institute of Health Economics and Health Care Management \& Munich Center of Health Sciences, Munich, Germany

Full list of author information is available at the end of the article
}

precise advice for patients with expectancy values for health outcomes and satisfaction thresholds is essential [7]. Thus, besides physician evaluation, a patient-based evaluation of generic and disease-specific health state changes by THR is necessary $[4,8]$. Therefore, EQ-5D and WOMAC (Western Ontario and McMaster Universities Arthritis Index) questionnaires are recognized as the most reliable, valid, and responsive in the literature $[9,10]$. Especially in the German context, it is not yet known if this applies to all patient groups and whether these patients are actually satisfied with usually positive health outcomes after THR. Thus, we analyze health outcomes and related satisfaction for the first time in a German prospective 
observation of a cohort. The current discussion on the benefit of THR can be supplemented by this health economics and medical analysis with patient-based generic quality of life (EQ-5D) and disease-specific function/ mobility/pain questionnaires (WOMAC). Patient characteristics and preoperative health scores are used to calculate the patient group-specific, expected value of THR. As our primary objective was to provide patient and clinician support in day-to-day routines of shared decision making, total knee replacement as a comparative procedure was not included in our analysis.

The integrative aspect of health outcome and satisfaction analysis gains importance for two reasons: (1) Shared decision making by patient and clinician - by using a personalized risk assessment approach - is becoming an important challenge to satisfy the patient [11]. This study gives patient group-specific information on average improvements in health outcomes and their time horizon to supply an empirical basis for shared decision making in THR. The study facilitates patient information and supports a patient's decision making in daily practice by comprehensive measures of health outcomes and satisfaction threshold values. To define the actual value of an intervention for the patient, and make it applicable to the patient, the practitioner, and health policy, an exact knowledge of health outcome drivers is necessary [12]. (2) This study calculates incremental effects for different patient subgroups to inform future intervention studies, costeffectiveness analyses, modeling approaches, or payment by results in THR. For comparative effectiveness research (CER) on osteoarthritis interventions, a grouping and distribution information for preoperative variables that affect health outcome is generated [13]. Thereby, we question gains in health outcomes for several patient groups.

We used a virtually exhaustive set of patient characteristics that have been shown previously to impact patient outcome in clinical studies [2], as well as information on common side diagnoses, comorbidities, and procedures. There is a research gap on the impact of preoperative patient characteristics on generic health outcomes, useful for understandable patient information and economic analysis of cost-effectiveness and benefit for defined patient groups, e.g., results inform about the probability of and time until a health state is better than before the operation.

The first aim of the study is to analyze the effect of preoperative patient characteristics on postoperative HRQoL, hip-specific pain, and function/mobility to be able to group patients for individual decision making based on the strongest predictors for health outcome changes. This facilitates patient information on surgery outcome and enables economic analysis of total hip replacement (THR) in different patient groups. To evaluate clinically relevant improvements and postoperative states the patient is satisfied with in daily practice, the patient acceptable symptom state (PASS) concept is used [14]. For THR, the PASS concept is a validated instrument in the literature $[15,16]$. It gives a threshold value beyond which patients define their health state as well or are actually satisfied with the results of THR [14]. 6 months after the operation patients reported health outcomes and were asked whether they were satisfied with THR. Satisfaction results were linked to their postoperative health outcome scores, to be able to support patients in day-to-day routines on their decision on THR with meaningful and appraisable expected health outcome changes from the perspective of the patient, the main objective of this study.

\section{Patients and methods Study design}

The study was designed as a single center, prospective observation of a cohort. Dependent variables were the change in HRQoL scores (EQ-5D) and hip-specific pain and function/mobility scores (WOMAC). The study used 6 months for follow-up, as prior studies have shown that most health improvements are reached within this period [15]. The 6-month time horizon is more imaginable and meaningful for a shared decision making situation with the patient than a later follow-up. However, this implies that the patient should be informed that the scores slightly underestimate the improvements by THA after a year or even three years as especially the function scores will still slightly improve after the 6-month time horizon. Predictive patient characteristics analyzed were separated into three subdomains: (1) socio-demographic factors; (2) medical factors; (3) HRQoL, pain, function, and mobility before THR (Table 1). This virtually exhaustive set of patient characteristics has been shown previously to impact patient outcome in clinical studies [2]. Essential for the observational study design was that all patients were treated similarly according to their major diagnosis, independent of their preoperative characteristics [17].

Recent studies refer to minimum clinically important differences (MCID) or patient acceptable symptom state (PASS) to define clinically relevant states for the patient $[7,14,18,19]$. As we want to define cut-off values of WOMAC or EQ-5D scores that are associated with patient satisfaction in THR and not only with a significant change in WOMAC or EQ-5D scores, two well established PASS methods were used: to calculate beyond which health state the patients are satisfied with THR outcome ("Were your expectations on THR fulfilled?"), we calculated PASS scores with the 75th centile method and the receiver operating characteristic (ROC) curve method [20]. Thereby, PASS is an anchor to provide more meaningful patient information and to help in interpreting health state results for patients and clinicians. 
Table 1 Predictive patient characteristics

Side diagnoses, at least 10 times in study patients

D62 - acute anemia* $\wedge^{\circ}$

E03 - hypothyroidism

E11 - diabetes ${ }^{* 0}$

E66 - obesity ${ }^{*} \wedge^{\circ}$

E78 - lipidemia

E79 - purine/pyrimidine metabolism

E86 - hypovolemia

E87 - dysfunction of water/electrolyte balance

F32 - depression

110 - arterial hypertonicity

125 - ischemic heart disease

148 - atrial fibrillation

J45 - asthma

N18/N39 - renal failure and related diseases

Z88 - drug allergy*

Z91 - risk factors in personal anamnesis*

Z92 - care of personal anamnesis ${ }^{\circ}$

Z95 - cardiac/vascular implants

Z96 - other functional implants ${ }^{\circ}$

n.n. - cardiopathy

n.n. - COPD

n.n. - hypercholesterolemia

n.n. - myocardial infarction/stent

n.n. - reflux^

n.n. - major hip distortion^

n.n. - deep venous thrombosis (DVT)

Number of side diagnoses*o

Operations and procedures, at least 5 times in study patients

5-791 - open reposition of fracture

5-829 - other arthroplasty*^

5-986 - minimally invasive technique

8-919 - acute pain relief

8-930/1 - monitoring

Number of operations and procedures*

\section{Other}

Age $^{\circ}$

Gender male* $\wedge^{\circ}$

$\mathrm{BMI}^{*}$

$\geq 30^{*} \wedge^{\circ}$

Major diagnosis ${ }^{\circ}$

Coxarthrosis 
Table 1 Predictive patient characteristics (Continued)

\begin{tabular}{|c|c|c|}
\hline \multicolumn{3}{|l|}{ Operations at joint before procedure ${ }^{\circ}$} \\
\hline 0 & 263 & 93.9 \\
\hline 1 & 12 & 4.3 \\
\hline 2 or more & 5 & 1.8 \\
\hline \multicolumn{3}{|l|}{ Marital status* $\wedge^{\circ}$} \\
\hline Married & 184 & 65.9 \\
\hline Single & 25 & 9.0 \\
\hline Divorced/living apart & 32 & 11.4 \\
\hline Widowed & 38 & 13.6 \\
\hline \multicolumn{3}{|l|}{ Housing situation*o } \\
\hline Alone & 79 & 28.4 \\
\hline With partner & 141 & 50.2 \\
\hline With family & 58 & 20.9 \\
\hline Discharge home (others inpatient rehabilitation) ${ }^{\circ}$ & 53 & 18.8 \\
\hline Health insurance compulsory (others private)*० & 136 & 48.4 \\
\hline Already $\mathrm{THR}^{\circ}$ & 58 & 20.6 \\
\hline Preoperative hemoglobin* $\wedge^{\circ}$ & $14.1(1.2)$ & \\
\hline Blood transfusion (yes)^ & 13 & 4.7 \\
\hline \multicolumn{3}{|l|}{ ASA score** } \\
\hline 1 & 109 & 38.8 \\
\hline 2 & 150 & 53.4 \\
\hline 3 or higher & 22 & 7.9 \\
\hline \multicolumn{3}{|l|}{ Charlson Comorbidity Index $\wedge^{*}{ }^{\circ}$} \\
\hline 0 & 210 & 74.4 \\
\hline 1 & 55 & 19.6 \\
\hline 2 & 9 & 3.2 \\
\hline 3 or higher & 7 & 2.5 \\
\hline Metabolic syndrome (yes)*o & 8 & 2.7 \\
\hline Cement (cement or hybrid)* & 33 & 11.8 \\
\hline
\end{tabular}

\section{Study population}

From January 2012 to June 2012, 387 patients at BBM Clinic participated in the health state evaluation before THR at the admission day and 321 also participated in the follow-up evaluation 6 months after discharge (Figure 1). Patients lost to follow-up did not differ significantly in most baseline characteristics. However, they had a statistically significantly lower preoperative WOMAC score $(-5.2)$ and EQ-5D score (-3.4). There were no exclusion criteria except missing patient consent. Besides patients with the major diagnosis of osteoarthritis of the hip, the study also included individuals with osteonecrosis. As we excluded revision THR, we had no mechanical complications or infections for major diagnoses. The study had approval from the ethics commission of Klinikum rechts der Isar, Technical University Munich.

\section{Measuring instruments}

According to clinical practice guidance, pain, function/ mobility, and HRQoL are limitations related to major

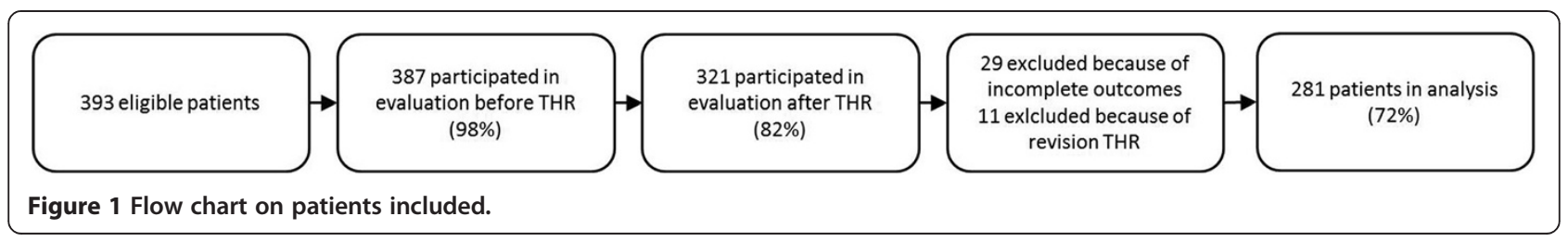


diagnoses leading to THR [21,22]. We measured HRQoL with the EQ-5D, a generic instrument that generates an index value with a standard formula out of five dimensions: mobility, self-care, usual activity, pain/discomfort, and anxiety/depression [23]. Each dimension has three possible levels to report current problems: no problems, some problems, and severe problems. To assign an overall value to each of the possible 253 health states, two standardized formulas are used $[23,24]$. To enable comparison with former studies and provide a basis for modeling purposes (e.g., for QALY calculation), we provide HRQoL outcomes based on the widely used UK population preference-weighted EQ-5D index values [24,25]. To respect actual experiences in the German population, we use a recently developed German population experienceweighted EQ-5D index [23]. The EQ-5D VAS that measures the overall health state was completed by the study population as the gold standard. The EQ-5D score is a qualified and well recognized health outcome measure in patients who receive THR: it has been tested for its validity, reliability, and responsiveness in relation to the SF-36, SF-6D, WOMAC, and Oxford Hip Score [9,26].

To measure hip-specific outcome from the patients' view, we use the WOMAC with its three subscales on pain (five sub-questions), stiffness (two sub-questions), mobility (17 sub-questions), and an overall score based on the three subscales. The sub-questions used a Likert scale from 0 to 10 . The questionnaire showed the best psychometric characteristics for hip and knee replacement patients in disease-specific questionnaires [10] and has also been tested for its validity in the German version $[27,28]$. We normalized each score into a $0-100$ scale, with 0 being the poorest and 100 being the best possible score, to improve comparability. Satisfaction with THR was measured using a Likert scale from 0 to 10 , with 9 and 10 defined as satisfied with THR. In the following we additionally provide change scores besides pre- and postoperative scores to support patient comprehension in day-to-day routines.

\section{Statistical analysis}

We provide descriptive statistics on the distribution of postoperative scores and corresponding change scores, and examined bivariate association with preoperative patient characteristics, using Spearman's rank correlation. In case Spearman's correlation is significant $(p<0.05)$, variables were considered as potential covariates for multivariate ordinary least squares (OLS) regression analyses. Statistical significance was assumed with $\mathrm{p}<0.05$. A power calculation (alpha $=0.05)$ for repeated measures of WOMAC and EQ-5D analyses showed an observed power of 1 . The final set of covariates was determined using a backward selection method based on significance in regression analysis. When comparing the health outcomes of preoperative EQ-5D and WOMAC groups, it has to be borne in mind that we use an observational study design where a regression to the mean effect is possible [29].

In determining clinically relevant health state changes (PASS), we were especially interested in the very satisfied group, as these estimates are not yet available and literature demands cut-off values for the highest levels of satisfaction [30] (Figure 2 and Figure 3). The ROC curve was used to identify satisfaction cut-off values for changes and 6-month postoperative WOMAC and EQ-5D scores. The ROC curve displays sensitivity/specificity pairs, where each corresponds to a possible cut-off value. We defined the optimal cut-off value where the difference in sensitivity and specificity is minimized. The area under the ROC curve (AUC) measures how well preoperative EQ-5D and WOMAC values separate between satisfied and unsatisfied patients. The closer the AUC is to 1 (the better it fills the upper triangles in Figure 3), the better EQ-5D and WOMAC can distinguish between satisfied and unsatisfied patients. Distribution functions of satisfied and unsatisfied patients were generated to show at which WOMAC or EQ-5D state their functions differentiate and to validate ROC curve results $[16,20]$ (75th centile method). The 75th centile method calculates health state values reached by $75 \%$ of the satisfied/unsatisfied patients (Figure 2).

\section{Results}

\section{Health outcomes}

The average age of patients was 68 years, $58 \%$ were female, and most patients had few general diseases (ASA 1 or 2 and Charlson Comorbidity Index 0 or 1). Some 21\% of the patients already had a hip replacement at the opposite side. Table 1 gives an overview of all control variables: significant associations with the changes and postoperative WOMAC and EQ-5D scores were observed for acute anemia, major hip distortion, BMI, preoperative hemoglobin, Charlson Comorbidity Index, etc. (Table 1).

Variance in health outcome changes was mainly explained by preoperative WOMAC and EQ-5D scores. A correlation matrix has shown high correlation of preoperative scores with score changes, 6-month follow-up scores, and weeks until a health state better than preoperative was achieved. Average WOMAC score and EQ-5D VAS before operation were 52 and 60 (0 worst, 100 best). Six months after THR, they had improved by 35 and 19 units (Figure 4 and Table 2). WOMAC and EQ-5D subscales improved accordingly. The poorer the preoperative WOMAC or any EQ-5D score, the higher the change in the scores. Patients with still acceptable preoperative scores had only slightly better postoperative scores compared with patients with poor preoperative WOMAC and EQ-5D scores (Figure 4 and Table 2). On average, $2 \%$ of THR patients described their health state, pain, and mobility as worse than before the procedure, about $6 \%$ described it as similar, $29 \%$ as better, and 

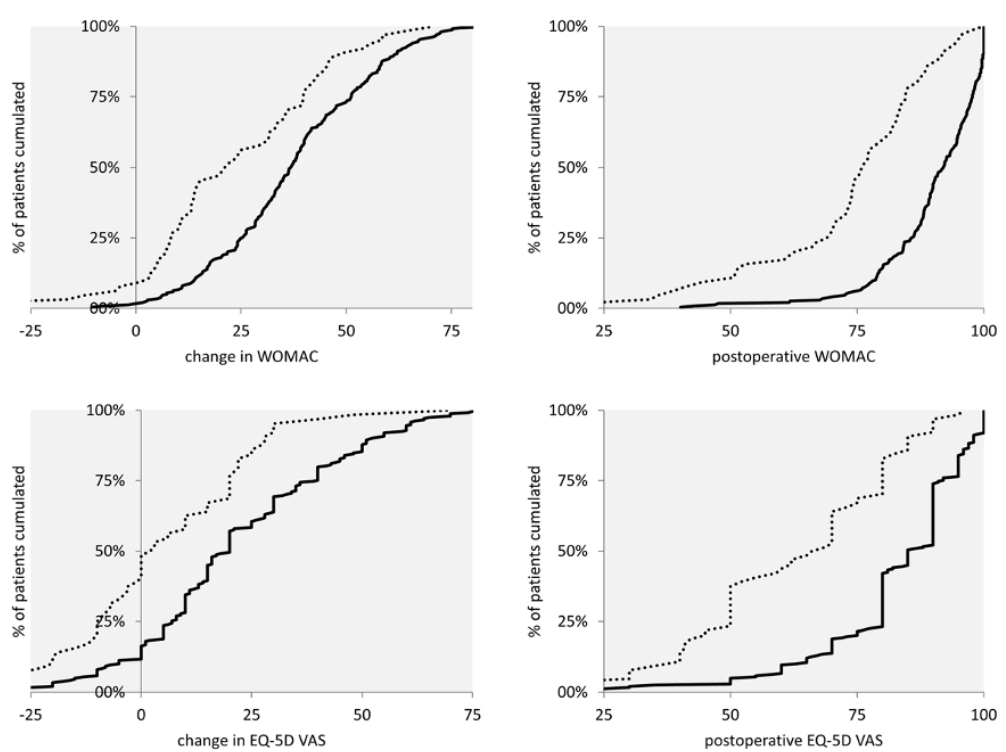

Figure 2 Distribution function to determine patient acceptable symptom state (PASS) with 75th centile method. Figure legend: ..... unsatisfied patients - - satisfied patients.

63\% as much better (Table 3). This description is highly correlated with WOMAC and all EQ-5D scores. Within EQ-5D dimensions, especially pain/discomfort, usual activity, and mobility improved for most patients (Additional file 1). On average, it takes 10 weeks until health state or daily routine is better than before the operation, 5 weeks until pain is better, and 8 weeks until mobility is better than before the operation (Table 3). The time until daily routine is better than before the operation decreases with a high preoperative WOMAC or EQ-5D score. With the patient characteristics analyzed, it was impossible to predict the time until pain or mobility was better than before the operation for different subgroups.

After multivariate regression analyses, we see that about $68 \%$ of the variance of changes in WOMAC and $47 \%$ of the variance of changes in EQ-5D VAS can be explained by very few preoperative patient characteristics: preoperative WOMAC and EQ-5D VAS scores, ASA score, metabolic syndrome, etc. (Table 4). When performing multivariate analysis with and without preoperative scores, we see that $75 \%$ of the explained variance is explained by the preoperative WOMAC score in the WOMAC change score model, and for the EQ-5D VAS change score model, $81 \%$ of the explained variance is explained by the preoperative EQ-5D VAS score. Thus, the change in WOMAC and EQ-5D can be well explained by preoperative WOMAC and EQ-5D only. Special risk groups or patient groups in which THR had no positive effect on WOMAC or EQ-5D scores could not be detected. Based on our statistical analyses, patients who do not benefit from THR cannot be identified by preoperative socio-demographic, medical, and health state characteristics.

\section{Satisfaction outcomes}

PASS estimates based on the ROC curve method and the 75th centile method were very similar. ROC curve estimates (Figure 3) of patients who considered their health state as satisfactory were above 85 for postoperative WOMAC (sensitivity 77\%, specificity $77 \%$ ), above 32 for change in WOMAC, above 79 for postoperative EQ-5D VAS (sensitivity $79 \%$, specificity $70 \%$ ), and above 14 for change in EQ-5D VAS. Sensitivity analyses on the satisfaction Likert scale, calculating cut-off values including 8, 9, and 10 or only 10 as the satisfactory state, show stable cutoff values. When only 10 is measured as satisfactory, cut-off values for 6-month postoperative states are 2 points higher; when 8 is included, cut-off values do not change. 75th centile estimates show similar results: of patients who considered their state satisfactory, $75 \%$ had a change in WOMAC of more than $25(\mathrm{CI} \pm 2)$. The satisfied patients had a postoperative WOMAC score above $86(\mathrm{CI} \pm 1)$. Concerning EQ$5 \mathrm{D}$ VAS change, $75 \%$ of the satisfied patients noted a change of 8 or higher $(\mathrm{CI} \pm 3)$. They had a postoperative EQ-5D VAS state of 80 or higher $(\mathrm{CI} \pm 3$ ) (Figure 2).

Socio-demographic and medical covariates for satisfied and unsatisfied patients did not vary with PASS values of both WOMAC and EQ-5D VAS, while preoperative WOMAC and EQ-5D VAS scores correlated with PASS $(p<0.01)[20,30]$. PASS outcomes show low probability of satisfaction for patients with WOMAC changes of less than 25 and EQ-5D VAS changes of less than 8. These patients have average preoperative WOMAC and EQ-5D VAS scores of 67 and 73 compared with 45 and 54 for the satisfied patients. Patients with postoperative WOMAC below 86 and EQ-5D VAS below 80 


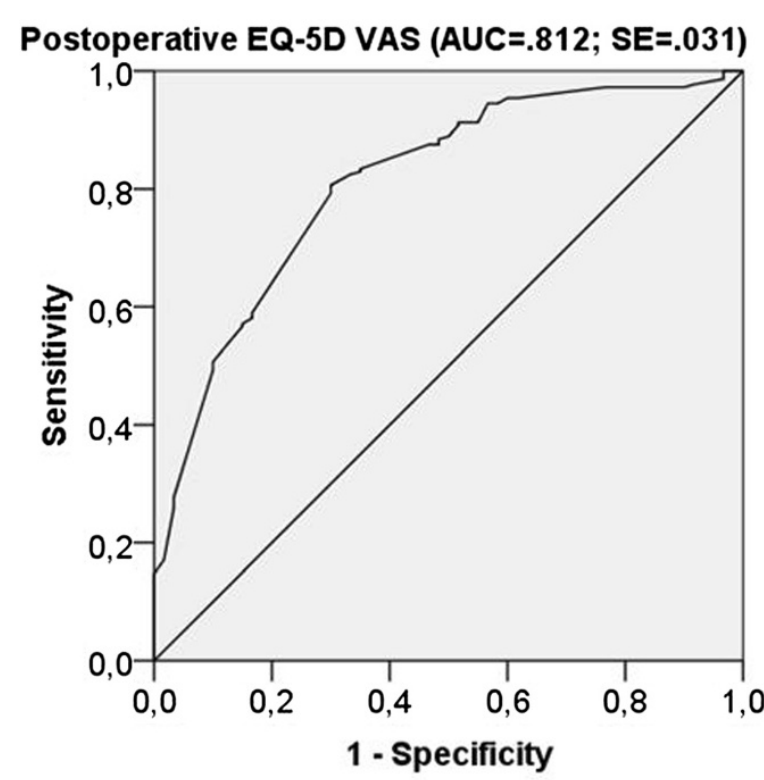

Postoperative WOMAC (AUC=.833; SE=.030)

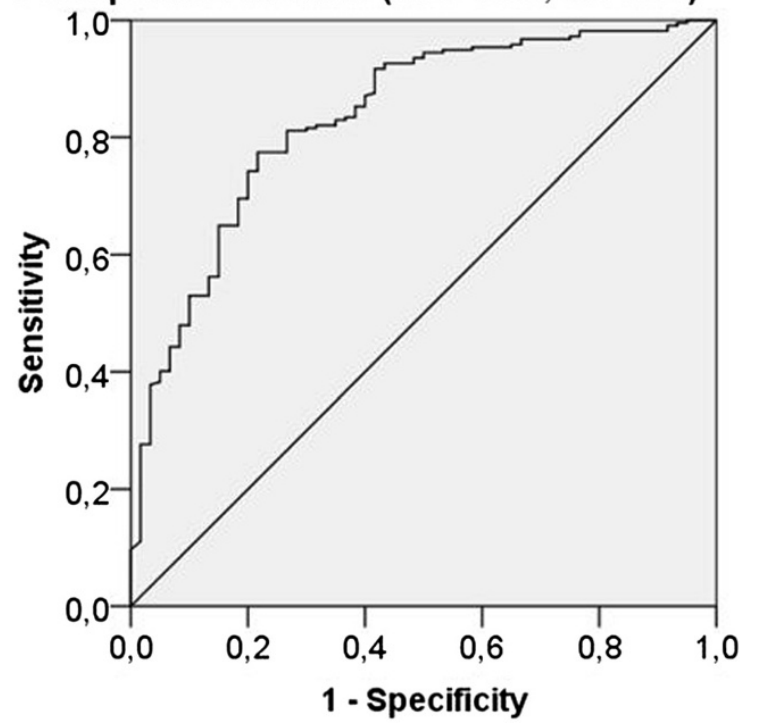

Figure 3 ROC curve using satisfaction and postoperative WOAMC/EQ-5D VAS scores.

also showed low probability of satisfaction. These patients have average preoperative WOMAC and EQ-5D VAS scores of 45 and 51 compared with 56 and 64 for the satisfied patients. This suggests that health outcome and preoperative health scores are related to satisfaction after THR: very poor and very good preoperative scores correlate with low satisfaction.

\section{Discussion}

\section{Health outcomes}

This study contributes to the literature of predictors of postoperative health outcomes after THR and the literature on predicting satisfaction after THR by investigating both subjects integrative in the German context. Related studies are by trend conform to our study [3,7,31-33]. THR outcome was mainly related to preoperative WOMAC and EQ-5D scores. There was a high correlation between WOMAC and EQ-5D scores, showing that the WOMAC results of THR patients can explain large parts of HRQoL, and that EQ-5D is a responsive instrument for THR patients. Although socio-demographic and medical covariates showed correlation with change and postoperative WOMAC and EQ-5D scores (Table 1), their contribution to the explanation was very low in multivariate models once the preoperative WOMAC and EQ-5D scores are accounted for (Table 4). Other studies were inconclusive on the impact of other preoperative variables than health state scores on postoperative health outcomes $[2,3]$. That predictability is highest with preoperative scores is in accordance with studies by Fortin et al., Röder et al., and Hawker et al. [7,32,34].

The separation of the most predictive variables (WOMAC and EQ-5D preoperatively) to support individual patient information and decision making showed that, for all groups, a significant improvement in WOMAC and HRQoL scores can be reached. The improvement is lowest in the group with still acceptable preoperative WOMAC scores (81-100) and acceptable preoperative EQ-5D scores (71100). According to clinical practice guidance, conservative therapy is useful when pain is still low and there are minor limitations in function/mobility and HRQoL. Afterwards there is an optimal time slot for THR where health outcomes and satisfaction are best. This conforms with the study results showing that patients with still acceptable preoperative WOMAC and EQ-5D scores have a tendency to benefit less from THR and also have a lower probability of being satisfied, as possible improvements from THR are lower (Figure 4). For patients with poorest scores, who also have a tendency to benefit less from THR, conservative therapy is no longer an option, questioning the use of THR only for patients in the group of still acceptable WOMAC or EQ-5D scores.

\section{Satisfaction outcomes}

In direct comparison to PASS results by Anakwe et al., Escobar et al., and Kvamme et al., we calculated a higher satisfaction threshold for EQ-5D and WOMAC scores as our baseline and follow-up scores of WOMAC and EQ-5D were higher $[15,35,36]$. Anakwe et al. showed that unlike postoperative scores, preoperative function scores are not related to satisfaction [35]. Several other studies found that WOMAC $[7,15,16,35,36]$ and EQ-5D [36] postoperative scores can be related to a PASS. As a prediction of PASS satisfaction measures to support patient's choice on THR does not yet exist, we used an indirect way to predict satisfaction for the patient preoperatively: we related satisfaction measures to postoperative and change 

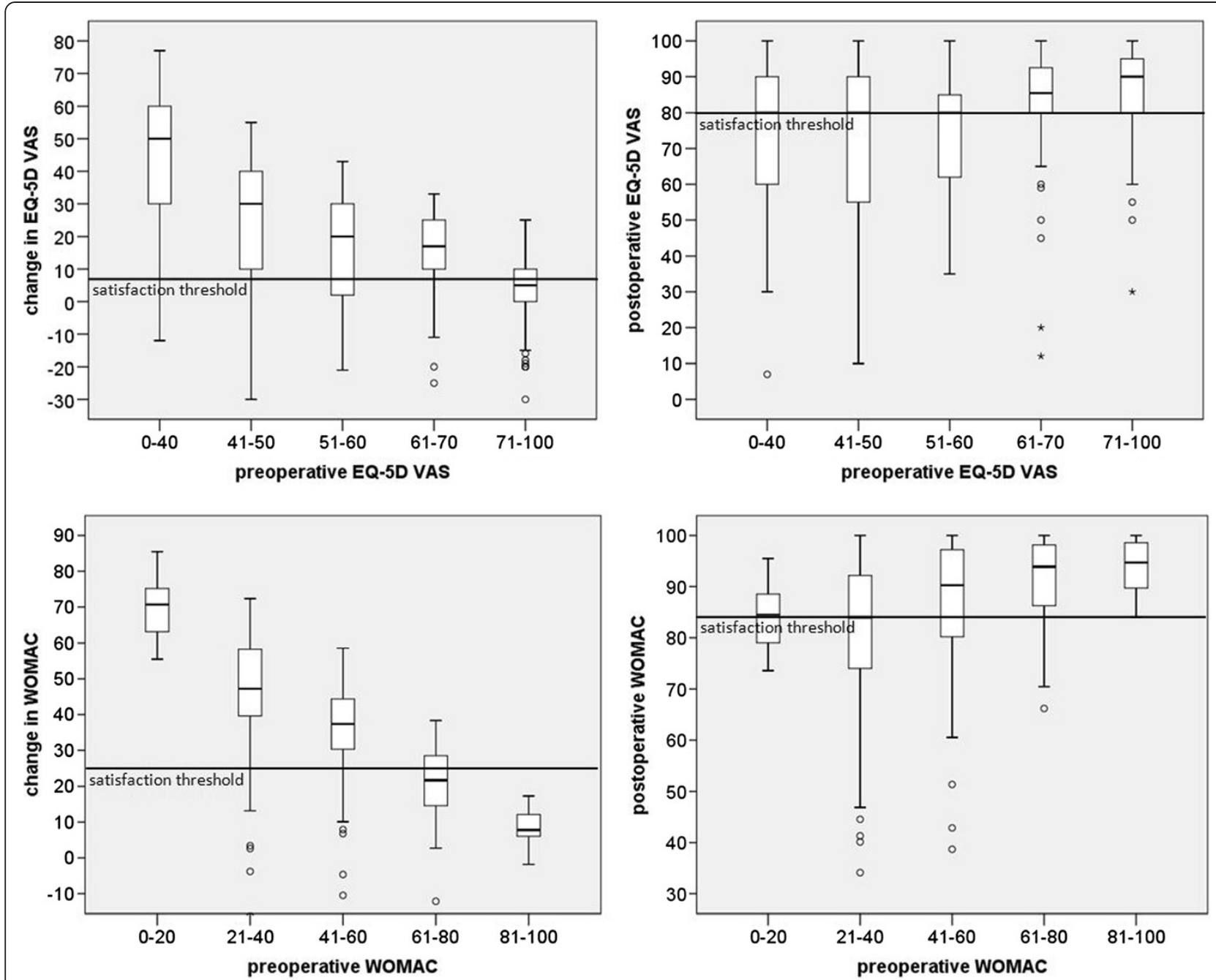

Figure 4 WOMAC sum and EQ-5D VAS boxplots. The horizontal lines correspond to the satisfaction thresholds (75th centile method).

Satisfaction is assumed at a 9 or 10 on a $0-10$ satisfaction Likert scale.

scores that can be expected by the classification of a patient into the given preoperative WOMAC and EQ-5D score groups.

The classification into preoperative EQ-5D and WOMAC groups and related satisfaction thresholds allows informed decision making on THR when included in preoperative patient information [30]. Presenting the patient's expected WOMAC and EQ-5D outcomes in a boxplot, separated by preoperative scores can facilitate patient choice when preoperative WOMAC and EQ-5D scores are calculated for the patient (Figure 4 and Additional file 2). In this study, satisfaction thresholds are given for the first time for the highest levels of satisfaction, and for WOMAC and EQ-5D changes and 6-month follow-up states. Besides this patient information, the introduction of standardized performance measures from a patient's perspective also has management relevance concerning marketing at the patient or practitioner level.

\section{Strengths and weaknesses}

Strengths of the study are the large patient group reached in a short period in a single hospital - allowing a distribution of the predictive factors - and the nearly exhaustive set of control variables analyzed simultaneously. Modelers benefit from preoperative EQ-5D and WOMAC distribution information. The distribution information on WOMAC and EQ-5D (Table 4) enables a transformation of WOMAC values into EQ-5D values, allowing (cost-)utility calculations with quality-adjusted life years (QALY) for studies that used WOMAC only. Another advantage is the conduct of the study in a standard supply hospital with a large catchment area to get a cross-section of patients and avoid preselection concerning, e.g., comorbidity [37]. On the other hand, generalizability of study results might not be given, as we used a single hospital to include only patients treated identically according to their medical need. Where applicable, patients were operated with minimally invasive 
Table 2 Changes in WOMAC and EQ-5D

\begin{tabular}{|c|c|c|c|c|c|c|c|}
\hline \multirow{2}{*}{$\begin{array}{l}0 \text { worst, } 100 \text { best } \\
\text { Preoperative }\end{array}$} & \multicolumn{2}{|c|}{ Pre-operative } & \multicolumn{2}{|c|}{6 months follow-up } & \multicolumn{2}{|c|}{ Change } & \multirow[b]{2}{*}{$\mathrm{N}$} \\
\hline & Mean & SE & Mean & SE & Mean & SE & \\
\hline & 55.6 & 1.1 & 90.4 & 0.8 & 34.7 & 1.2 & 281 \\
\hline $0-20$ & 12.9 & 1.3 & 89.0 & 2.1 & 76.1 & 2.2 & 14 \\
\hline $21-40$ & 33.1 & 0.8 & 85.7 & 2.4 & 52.6 & 2.6 & 45 \\
\hline $41-60$ & 51.4 & 0.5 & 87.8 & 1.4 & 36.4 & 1.4 & 110 \\
\hline $61-80$ & 70.8 & 0.6 & 94.9 & 0.8 & 24.1 & 1.0 & 89 \\
\hline $81-100$ & 87.7 & 1.0 & 95.3 & 1.2 & 7.7 & 1.6 & 23 \\
\hline WOMAC stiffness & 46.6 & 1.4 & 83.0 & 1.0 & 36.5 & 1.5 & 281 \\
\hline $0-20$ & 14.1 & 0.8 & 79.1 & 2.5 & 65.0 & 2.7 & 56 \\
\hline $21-40$ & 33.2 & 0.7 & 81.1 & 2.2 & 48.0 & 2.3 & 66 \\
\hline $41-60$ & 53.4 & 0.6 & 82.2 & 2.0 & 28.8 & 2.0 & 86 \\
\hline $61-80$ & 71.9 & 0.8 & 87.8 & 1.6 & 15.8 & 1.9 & 60 \\
\hline $81-100$ & 91.9 & 1.3 & 93.1 & 3.1 & 1.2 & 2.5 & 13 \\
\hline WOMAC function & 51.3 & 1.1 & 86.7 & 0.8 & 35.5 & 1.1 & 281 \\
\hline $0-20$ & 16.4 & 1.0 & 79.4 & 3.1 & 63.4 & 3.4 & 21 \\
\hline $21-40$ & 33.6 & 0.6 & 78.8 & 2.1 & 45.3 & 2.2 & 69 \\
\hline $41-60$ & 50.5 & 0.6 & 88.1 & 1.2 & 37.5 & 1.3 & 96 \\
\hline $61-80$ & 69.4 & 0.6 & 92.2 & 0.8 & 22.8 & 1.1 & 77 \\
\hline $81-100$ & 85.9 & 1.0 & 94.9 & 1.2 & 9.0 & 1.3 & 18 \\
\hline WOMAC sum & 51.8 & 1.1 & 86.7 & 0.8 & 34.9 & 1.1 & 281 \\
\hline $0-20$ & 15.2 & 1.4 & 84.2 & 1.7 & 69.0 & 2.2 & 14 \\
\hline $21-40$ & 33.7 & 0.6 & 79.6 & 2.1 & 45.9 & 2.2 & 71 \\
\hline $41-60$ & 51.2 & 0.6 & 87.4 & 1.2 & 36.2 & 1.3 & 108 \\
\hline $61-80$ & 70.3 & 0.7 & 91.6 & 1.0 & 21.3 & 1.2 & 73 \\
\hline $81-100$ & 85.4 & 0.9 & 93.9 & 1.3 & 8.5 & 1.3 & 15 \\
\hline EQ-5D index* & 53.9 & 0.9 & 76.9 & 0.9 & 22.9 & 1.0 & 281 \\
\hline $0-40$ & 33.1 & 0.5 & 70.1 & 2.1 & 37.1 & 2.1 & 69 \\
\hline $41-50$ & 46.8 & 0.3 & 76.2 & 2.2 & 29.3 & 2.1 & 41 \\
\hline $51-60$ & 54.2 & 0.2 & 76.4 & 1.7 & 22.2 & 1.8 & 70 \\
\hline $61-70$ & 65.5 & 0.3 & 80.7 & 1.4 & 15.3 & 1.5 & 53 \\
\hline $71-100$ & 76.9 & 0.6 & 83.5 & 1.4 & 6.7 & 1.6 & 48 \\
\hline EQ-5D index ${ }^{* *}$ & 51.4 & 1.8 & 84.7 & 1.1 & 33.3 & 1.8 & 281 \\
\hline-40 & 8.8 & 1.1 & 79.4 & 2.5 & 70.5 & 2.3 & 88 \\
\hline $41-50$ & - & - & - & - & - & - & - \\
\hline $51-60$ & 56.8 & 0.6 & 85.4 & 3.3 & 28.6 & 3.0 & 26 \\
\hline $61-70$ & 66.6 & 0.3 & 84.3 & 1.8 & 17.7 & 1.9 & 73 \\
\hline $71-100$ & 77.9 & 0.6 & 89.8 & 1.3 & 12.0 & 1.4 & 94 \\
\hline EQ-5D VAS & 59.6 & 1.2 & 78.4 & 1.1 & 18.8 & 1.4 & 281 \\
\hline $0-40$ & 28.0 & 1.3 & 74.7 & 2.8 & 46.7 & 2.6 & 57 \\
\hline $41-50$ & 48.3 & 0.4 & 70.1 & 3.4 & 21.8 & 3.4 & 49 \\
\hline $51-60$ & 58.4 & 0.5 & 74.1 & 2.8 & 15.7 & 2.9 & 37 \\
\hline $61-70$ & 68.8 & 0.3 & 81.5 & 2.5 & 12.8 & 2.6 & 52 \\
\hline $71-100$ & 81.9 & 0.8 & 85.4 & 1.3 & 3.5 & 1.3 & 86 \\
\hline
\end{tabular}

*German population experience-weighted.

**UK population preference-weighted. 
Table 3 Weeks until effect was reached and general effect 6 months postoperatively

\begin{tabular}{|c|c|c|c|c|c|c|}
\hline Weeks until effect was reached & Daily routine & & Pain & & Mobility & \\
\hline Weeks mean & 9.6 & & 5.1 & & 8.1 & \\
\hline Weeks SD & 5.4 & & 4.9 & & 5.2 & \\
\hline Not yet better & $6.0 \%$ & & $4.9 \%$ & & $5.6 \%$ & \\
\hline Effect 6 month postoperatively & Frequency & $\%$ & Frequency & $\%$ & Frequency & $\%$ \\
\hline Much worse & 0 & 0.0 & 0 & 0.0 & 3 & 1.1 \\
\hline Worse & 7 & 2.6 & 3 & 1.1 & 5 & 1.8 \\
\hline Similar & 21 & 7.7 & 7 & 2.6 & 18 & 6.6 \\
\hline Better & 90 & 32.8 & 50 & 18.3 & 98 & 35.8 \\
\hline Much better & 156 & 56.9 & 213 & 78.0 & 150 & 54.7 \\
\hline
\end{tabular}

technique. With patients needing uncemented arthroplasty the Allofit cup/Spotorno stem system by Zimmer was used as the standard, for hybrid systems a similar cup and a Müller Straight stem by Zimmer was used, and for cemented systems a Müller PE cup and a Müller Straight stem was used as the standard. Case numbers for other arthroplasty techniques than minimally invasive or for prosthesis types were too low in the subgroups to find statistically significant differences.

Weaknesses of the study are: (1) the low patient number in some subgroups that did, e.g., not allow to go into a further detailed analysis of operation techniques and prosthesis type used; (2) that socio-economic variables were limited to compulsory and private health insurance, differentiating roughly between high and low income patients. Here we would expect differences in socio-economic groups, as a difference in health outcome between compulsory and private insurance could already be detected; (3) that more patients with lower a health state did not participate in follow-up, although a lower preoperative health state in WOMAC and EQ-5D is related to a higher than average health improvement; (4) p-values in Table 4 are of restricted validity as we had to specify and estimate on the same dataset - a split-sample design was not

Table 4 Multivariate linear regression

\begin{tabular}{|c|c|c|c|c|}
\hline \multirow{3}{*}{$\begin{array}{l}\text { Preoperative values } \\
{ }^{*} \mathrm{p}<.05 * * \mathrm{p}<.01\end{array}$} & \multirow{2}{*}{\multicolumn{2}{|c|}{$\begin{array}{l}\text { Change in WOMAC sum } \\
\left.\text { (adj. } R^{2}=0.68\right)\end{array}$}} & \multirow{2}{*}{\multicolumn{2}{|c|}{$\begin{array}{l}\text { Change in EQ-5D VAS } \\
\left.\text { (adj. } R^{2}=0.47\right)\end{array}$}} \\
\hline & & & & \\
\hline & Coefficient & SE & Coefficient & SE \\
\hline Constant & **98.307 & 6.455 & **77.959 & 10.141 \\
\hline WOMAC pain score & **-0.166 & .062 & -.189 & .099 \\
\hline WOMAC stiffness score & **-0.191 & .042 & & \\
\hline WOMAC function score & $* *-0.536$ & .068 & .185 & .101 \\
\hline EQ-5D usual activity severe problems compared to none & $* *-47.200$ & 11.407 & & \\
\hline EQ-5D anxiety/depression some problems compared to none & $* *-4.454$ & 1.598 & $*-5.820$ & 2.563 \\
\hline EQ-5D visual analog scale (VAS) & & & **-0.797 & .064 \\
\hline major hip distortion & -4.706 & 2.797 & -8.306 & 4.462 \\
\hline E11 - diabetes & -6.033 & 3.108 & & \\
\hline E66 - obesity & 5.735 & 3.247 & & \\
\hline Z96 - other functional implants & *-3.896 & 1.946 & & \\
\hline 5-829 - other arthroplasty & & & -14.422 & 7.363 \\
\hline Reflux & $* *-8.502$ & 2.901 & & \\
\hline Number of operations and procedures & -1.277 & .762 & & \\
\hline Housing situation family compared to alone & -18.877 & 11.151 & & \\
\hline Discharge home compared to inpatient rehabilitation & 3.537 & 1.831 & & \\
\hline ASA 2 compared to 1 & $* *-4.611$ & 1.501 & & \\
\hline Discharge home compared to inpatient rehabilitation & & & *7.113 & 2.789 \\
\hline Metabolic syndrome & **-13.752 & 5.147 & $* *-22.776$ & 6.432 \\
\hline
\end{tabular}


reasonable due to the limited number of cases and would have supported validity of results; and (5) we did not calculate special PASS values for patients in each preoperative WOMAC and EQ-5D index score group besides the overall PASS score, as we did not have enough observations in each category to calculate a special PASS value in each group with either the ROC curve method [16] or the 75th centile method [14]. By getting similar PASS cut-off values with both methods and similar cut-off values within a sensitivity analysis for the satisfaction measure, we expect to have robust results for the satisfaction thresholds of WOMAC and EQ-5D. However, as patients with different preoperative health states might have different perceptions on their satisfaction threshold, this subgroup analysis should be researched in the future.

\section{A future perspective}

Change and postoperative HRQoL and WOMAC scores are useful performance or patient value measures that can be used as quality indicators in pay for performance systems [12]. In case WOMAC and EQ-5D change scores are used as additional influencing factors for performancerelated reimbursement, the WOMAC is the preferred instrument, as it is less influenced by comorbidities not related to THR and has higher correlation with the preoperative score. Future research should match pre- and postoperative EQ-5D values with population normative values to further quantify the utility of THR for each preoperative EQ-5D group. The study allows a combination of HRQoL measures with costing data at patient level, supporting national and international comparison of effects and cost-effectiveness based on preoperative EQ-5D scores. By giving incremental effects for different patient subgroups, we support future comparative effectiveness research on osteoarthritis interventions and across countries. The National Health Service (NHS) in England has already introduced the EQ-5D questionnaire as a standardized questionnaire for a patient-reported outcome measure (PROM), to be able to measure hospital performance and cost-effectiveness for patient groups. A comparison of EQ5D changes with English values [38] on a patient basis can show how routine data collection of health outcomes can be introduced in further countries. To provide patients not only with short term expectations (6 months) on health outcomes, quality of life, and satisfaction, a second followup period at 24 or 36 month should be provided for long term expectations as especially function scores still slightly improve after 6 months [2].

\section{Conclusions}

Changes in WOMAC and EQ-5D scores can mainly be explained by preoperative scores. Other covariates contributed only marginally to predict THR health outcomes. Based on the PASS concept, very good or very poor preoperative scores lower the probability of patient satisfaction with THR. However, a patient group in which HRQoL or WOMAC scores could not be improved or a patient group that was unsatisfied could not be detected. THR should not be restricted to subgroups, as all subgroups can benefit. Shared decision making using a personalized risk assessment approach provides predicted health states and satisfaction thresholds to patients and can support the individual decision on THR.

\section{Additional files}

\section{Additional file 1: Changes in EQ-5D dimensions. \\ Additional file 2: Slide on expected WOMAC and EQ-5D outcomes for German patients.}

\section{Abbreviations}

ASA: American Society of Anesthesiologists; CER: Comparative effectiveness research; EQ-5D: EuroQol 5-dimension; EQ-5D VAS: EuroQol 5-dimension visual analogue scale; HRQoL: Health-related quality of life; OLS: Ordinary least squares; THR: Total hip replacement; PASS: Patient acceptable symptom state; PROMs: Patient-reported outcome measures; NHS: National Health Service; QALY: Quality-adjusted life year; ROC: Receiver operating characteristic; WOMAC: Western Ontario and McMaster Universities Arthritis Index.

\section{Competing interests}

The authors declare that they have no competing interests.

\section{Authors' contributions}

MV and WP designed the study and drafted the manuscript. RW and CL carried out the study and helped to draft the manuscript. MH supported the statistical analysis and helped to draft the manuscript. All authors read and approved the final manuscript.

\section{Author details}

${ }^{1}$ Helmholtz Zentrum München, German Research Center for Environmental Health, Institute of Health Economics and Health Care Management, P.O. Box 1129, Neuherberg 85758, Germany. ${ }^{2}$ Ludwig-Maximilians-Universität München, Munich School of Management, Institute of Health Economics and Health Care Management \& Munich Center of Health Sciences, Munich, Germany. ${ }^{3}$ Krankenhaus Barmherzige Brüder München, Akademisches Lehrkrankenhaus der Technischen Universität München, Romanstraße 93, Munich 80639, Germany. ${ }^{4}$ Klinikum rechts der Isar, Technical University Munich, Munich, Germany.

Received: 3 February 2014 Accepted: 24 June 2014

Published: 7 August 2014

\section{References}

1. Laupacis A, Bourne R, Rorabeck C, Feeny D, Wong C, Tugwell P, Leslie K, Bullas $R$ : The effect of elective total hip replacement on health-related quality of life. J Bone Joint Surg Am 1993, 75:1619-1626.

2. Ethgen O, Bruyere O, Richy F, Dardennes C, Reginster JY: Health-related quality of life in total hip and total knee arthroplasty. A qualitative and systematic review of the literature. J Bone Joint Surg Am 2004, 86-A:963-974.

3. Biring GS, Masri BA, Greidanus NV, Duncan CP, Garbuz DS: Predictors of quality of life outcomes after revision total hip replacement. J Bone Joint Surg (Br) 2007, 89:1446-1451.

4. Learmonth ID, Young C, Rorabeck C: The operation of the century: total hip replacement. Lancet 2007, 370:1508-1519.

5. Kurtz S, Ong K, Lau E, Mowat F, Halpern M: Projections of primary and revision hip and knee arthroplasty in the United States from 2005 to 2030. J Bone Joint Surg Am 2007, 89:780-785. 
6. OECD: Health at a glance Europe. 2012, [http://www.oecd.org/els/healthsystems/HealthAtAGlanceEurope2012.pdf]

7. Hawker GA, Badley EM, Borkhoff CM, Croxford R, Davis AM, Dunn S, Gignac $M A$, Jaglal SB, Kreder HJ, Sale JE: Which patients are most likely to benefit from total joint arthroplasty? Arthritis Rheum 2013, 65:1243-1252.

8. Stratford PW, Kennedy DM: Performance measures were necessary to obtain a complete picture of osteoarthritic patients. J Clin Epidemiol 2006, 59:160-167.

9. Ostendorf M, van Stel HF, Buskens E, Schrijvers AJ, Marting LN, Verbout AJ, Dhert WJ: Patient-reported outcome in total hip replacement. A comparison of five instruments of health status. J Bone Joint Surg (Br) 2004, 86:801-808.

10. Veenhof C, Bijlsma JW, van den Ende CH, van Dijk GM, Pisters MF, Dekker J: Psychometric evaluation of osteoarthritis questionnaires: a systematic review of the literature. Arthritis Rheum 2006, 55:480-492.

11. Alambuya RN, Ali S, Apostolidis K, Beinfeld MT, Bijwe SR, Bravo P, Brocklehurst W, Davis R, Dhir A, Dodd CR, Durand MA, Eaton S, Faber M, Fisler CM, Ford PA, Westrick MG, Nikovska DG, Hendy MA, Johnson C, Johnstone R, Laitner S, Lam LS, Lee DM, Lynch HF, Mankeekar PC, Mbaabu LM, McCay L, Monti M, Moulton BW, Muthambi TC, et al: Salzburg statement on shared decision making. BMJ 2011, 342:d1745.

12. Porter ME: What is value in health care? N Engl J Med 2010, 363:2477-2481.

13. Sorenson C, Gusmano MK, Oliver A: The politics of comparative effectiveness research: lessons from recent history. J Health Polit Policy Law 2013, [Epub ahead of print].

14. Tubach F, Ravaud P, Baron G, Falissard B, Logeart I, Bellamy N, Bombardier C, Felson D, Hochberg M, van der Heijde D, Dougados M: Evaluation of clinically relevant states in patient reported outcomes in knee and hip osteoarthritis: the patient acceptable symptom state. Ann Rheum Dis 2005, 64:34-37.

15. Escobar A, Gonzalez M, Quintana JM, Vrotsou K, Bilbao A, Herrera-Espineira C, Garcia-Perez L, Aizpuru F, Sarasqueta C: Patient acceptable symptom state and OMERACT-OARSI set of responder criteria in joint replacement. Identification of cut-off values. Osteoarthritis Cartilage 2012, 20:87-92.

16. Arden NK, Kiran A, Judge A, Biant LC, Javaid MK, Murray DW, Carr A , Cooper C, Field RE: What is a good patient reported outcome after total hip replacement? Osteoarthritis Cartilage 2011, 19:155-162.

17. Altman DG: Systematic reviews of evaluations of prognostic variables. BMJ 2001, 323:224-228.

18. Chesworth BM, Mahomed NN, Bourne RB, Davis AM: Willingness to go through surgery again validated the WOMAC clinically important difference from THR/TKR surgery. J Clin Epidemiol 2008, 61:907-918.

19. Singh J, Sloan JA, Johanson NA: Challenges with health-related quality of life assessment in arthroplasty patients: problems and solutions. J Am Acad Orthop Surg 2010, 18:72-82.

20. Kvien TK, Heiberg T, Hagen KB: Minimal clinically important improvement/ difference (MCII/MCID) and patient acceptable symptom state (PASS): what do these concepts mean? Ann Rheum Dis 2007, 66 Suppl 3:iii40-41.

21. Deutsche Gesellschaft für Unfallchirurgie: Leitlinien der deutschen gesellschaft für unfallchirurgie - endoprothese bei koxarthrose. [http://www.awmf.org/uploads/tx_szleitlinien/012-006l_S1_Endoprothese_ bei_Koxarthrose_2008.pdf]

22. National Institute for Health and Clinical Excellence (NICE): Osteoarthritis she care and management of osteoarthritis in adults. [http://www.nice. org.uk/nicemedia/pdf/cg59niceguideline.pdf]

23. Leidl R, Reitmeir P: A value set for the EQ-5D based on experienced health states: development and testing for the German population. Pharmacoeconomics 2011, 29:521-534.

24. Dolan P: Modelling valuations for health states: the effect of duration. Health Policy 1996, 38:189-203.

25. Whitehead SJ, Ali S: Health outcomes in economic evaluation: the QALY and utilities. Br Med Bull 2010, 96:5-21.

26. Barton GR, Sach TH, Avery AJ, Doherty M, Jenkinson C, Muir KR: Comparing the performance of the EQ-5D and SF-6D when measuring the benefits of alleviating knee pain. Cost Eff Resour Alloc 2009, 7:12.

27. Stucki G, Meier D, Stucki S, Michel BA, Tyndall AG, Dick W, Theiler R: [Evaluation of a German version of WOMAC (Western Ontario and McMaster Universities) Arthrosis Index]. Z Rheumatol 1996, 55:40-49

28. Roos EM, Klassbo M, Lohmander LS: WOMAC osteoarthritis index. Reliability, validity, and responsiveness in patients with arthroscopically assessed osteoarthritis. Western Ontario and MacMaster Universities. Scand J Rheumatol 1999, 28:210-215.

29. Van Breukelen GJ: ANCOVA versus change from baseline: more power in randomized studies, more bias in nonrandomized studies [corrected]. J Clin Epidemiol 2006, 59:920-925

30. Judge A, Arden NK, Kiran A, Price A, Javaid MK, Beard D, Murray D, Field RE: Interpretation of patient-reported outcomes for hip and knee replacement surgery: identification of thresholds associated with satisfaction with surgery. J Bone Joint Surg (Br) 2012, 94:412-418.

31. Davis AM, Agnidis Z, Badley E, Kiss A, Waddell JP, Gross AE: Predictors of functional outcome two years following revision hip arthroplasty. J Bone Joint Surg Am 2006, 88:685-691.

32. Fortin PR, Clarke AE, Joseph $L$, Liang MH, Tanzer M, Ferland D, Phillips $C$, Partridge AJ, Bélisle P, Fossel AH, Mahomed N, Sledge CB, Katz JN: Outcomes of total hip and knee replacement: preoperative functional status predicts outcomes at six months after surgery. Arthritis Rheum 1999, 42:1722-1728.

33. Nilsdotter AK, Petersson IF, Roos EM, Lohmander LS: Predictors of patient relevant outcome after total hip replacement for osteoarthritis: a prospective study. Ann Rheum Dis 2003, 62:923-930.

34. Roder C, Staub LP, Eggli S, Dietrich D, Busato A, Muller U: Influence of preoperative functional status on outcome after total hip arthroplasty. J Bone Joint Surg Am 2007, 89:11-17.

35. Anakwe RE, Jenkins PJ, Moran M: Predicting dissatisfaction after total hip arthroplasty: a study of 850 patients. J Arthroplasty 2011, 26:209-213.

36. Kvamme MK, Kristiansen IS, Lie E, Kvien TK: Identification of cutpoints for acceptable health status and important improvement in patient-reported outcomes, in rheumatoid arthritis, psoriatic arthritis, and ankylosing spondylitis. J Rheumatol 2010, 37:26-31.

37. Schafer T, Pritzkuleit R, Jeszenszky C, Malzahn J, Maier W, Gunther KP, Niethard F: Trends and geographical variation of primary hip and knee joint replacement in Germany. Osteoarthritis Cartilage 2013, 21:279-288.

38. Gutacker N, Bojke C, Daidone S, Devlin N, Street A: Hospital variation in patient-reported outcomes at the level of EQ-5D dimensions: evidence from England. Med Decis Making 2013, 33:804-818.

doi:10.1186/s12955-014-0108-1

Cite this article as: Vogl et al:: The impact of preoperative patient characteristics on health states after total hip replacement and related satisfaction thresholds: a cohort study. Health and Quality of Life Outcomes 2014 12:108.

\section{Submit your next manuscript to BioMed Central and take full advantage of:}

- Convenient online submission

- Thorough peer review

- No space constraints or color figure charges

- Immediate publication on acceptance

- Inclusion in PubMed, CAS, Scopus and Google Scholar

- Research which is freely available for redistribution 